\title{
VERIFICAÇÃO DA EFICIÊNCIA DA INSPEÇÃO VIRTUAL DE MANIFESTAÇÕES PATOLÓGICAS EM FACHADAS DE EDIFÍCIOS COM DIFERENTES REVESTIMENTOS
}

\author{
PISTUNI SOLANHO, BIANCA \\ Engenheira Civil \\ Universidade do Vale do Itajaí \\ Santa Catarina; Brasil \\ bsolanho@gmail.com \\ VARGAS, JAMILLE \\ Engenheira Civil \\ Universidade do Vale do Itajaí \\ Santa Catarina; Brasil \\ jamille@construtoracopas.com.br \\ MORESCO SILVA, JULIANO \\ Engenheiro Civil \\ Universidade do Sul de Santa Catarina \\ Santa Catarina; Brasil \\ julianomoresco@gmail.com
}

\author{
RAMALHO PEREIRA TASCA, FERNANDA \\ Engenheira Civil \\ Centro Universitário Católica de Santa Catarina \\ Santa Catarina; Brasil \\ tascafernandatasca@gmail.com
}

\author{
BITTENCOURT FORTE, LAÍS \\ Arquiteta e Urbanista \\ Universidade do Sul de Santa Catarina \\ Santa Catarina; Brasil \\ laisbitten@gmail.com
}

\author{
LUIZ STOCCO, JOELCIO \\ Engenheiro Civil \\ Universidade Federal de Santa Catarina \\ Santa Catarina; Brasil \\ eng.stocco@gmail.com
}

\section{RESUMO}

As fachadas são definidas como a camada de proteção das edificações, necessitando de um cuidado especial através da realização de inspeções e manutenções. No Brasil ainda não há normas relacionadas à inspeção de fachadas, apenas um "projeto lei", ainda sem votação, que prevê inspeções periódicas. A metodologia mais conhecida é a inspeção física, onde o profissional vai até o problema com o auxílio de um equipamento de movimentação vertical externa. Com a tecnologia, há um novo método conhecido como inspeção virtual, onde utiliza-se um equipamento com uma câmera de alta resolução que capta imagens aéreas (drone), podendo ser realizado por um único profissional, estático em solo, apenas manuseando o equipamento. Neste estudo o intuito foi comparar a eficiência da inspeção virtual com a inspeção física em fachadas com diferentes tipos de revestimentos e também verificar a viabilidade econômica. Definiu-se uma edificação e, após a elaboração dos métodos de inspeção, o procedimento foi baseado em duas etapas: física e virtual. Ao final, as manifestações patológicas observadas foram quantificadas e classificadas para posterior comparação entre os diferentes métodos. Nas fachadas revestidas com pintura, os dois procedimentos se assemelharam na eficiência e precisão dos resultados, sendo que a inspeção virtual apresentou maior rapidez para o registro, além da vantagem de revisão das imagens. Já nas fachadas revestidas com placas cerâmicas, o procedimento virtual não possibilitou a detecção de danos como desplacamentos, visto que seria necessário teste físico. Por fim, verificou-se maior viabilidade econômica para a inspeção virtual devido à correlação entre menor custo do aluguel do drone e horas trabalhadas.

Palavras-chave: drone, fachada, inspeção, manifestação patológica.

\section{ABSTRACT}

Facades are defined as the protection layer of buildings, requiring special care through inspections and maintenance. In Brazil there are no standards related to the inspection of facades, just a "bill", still without voting, which provides for periodic inspections. The best known methodology is physical inspection, where the professional goes to the problem with the help of an external vertical movement equipment. With technology, there is a new method known as virtual inspection, which uses a device with a high resolution camera that captures aerial images (drone), can be performed by a single professional, static on the ground, just handling the equipment. The purpose of this study was to compare the efficiency of virtual inspection with physical inspection on facades with different types of cladding and also to verify the economic viability. A building was defined and, after the elaboration of the inspection methods, the procedure was based on two steps: physical and virtual. At the end, the observed pathological manifestations were quantified and classified for later comparison between the different methods. In paint-coated facades, the two procedures were similar in efficiency and precision of results, and the virtual inspection showed faster registration, besides the advantage of 
reviewing the images. In the facades covered with ceramic plates, the virtual procedure did not allow the detection of damage such as slippage, since it would be necessary to perform a physical test. Finally, there was greater economic viability for virtual inspection due to the correlation between lower drone rental cost and hours worked. Keywords: drone, facade, inspection, pathological manifestation.

\section{INTRODUÇÃO}

As fachadas são definidas como a camada de proteção das edificações, tendo como principal função a de vedação. Devido ao fato de serem um dos sistemas mais afetados pelas intempéries, poluição, contaminação química e variações de temperatura, nota-se o crescente número de edificações com desempenho insatisfatório no Brasil (CONSOLI, 2006; GOLDBERG, 1998).

Um estudo realizado pelo IBAPE-SP (2015), apontou que $66 \%$ das prováveis causas e origens dos acidentes provocados pela presença de manifestações patológicas estão relacionados à deficiência e inexistência de manutenção predial. A prática da realização de inspeções periódicas nas edificações e também da manutenção preventiva, reduz a ocorrência de acidendes e é indispensável para garantir que se atinja a vida útil mínima das edificações (ALVES, 2009).

Tratando-se das inspeções de fachadas, não há normas no Brasil, apenas um "projeto lei”, ainda sem votação, que prevê inspeções periódicas para avaliar o grau de risco à segurança dos usuários (IBAPE-RS, 2012). Então, atualmente os profissionais têm-se embasado apenas em algumas normas da Associação Brasileira de Normas Técnicas (ABNT), como: NBR 13752 (ABNT, 1996), que especifica os critérios e procedimentos para a realização de perícias; NBR 15575 (ABNT, 2013), que aborda critérios e níveis de desempenho para os sistemas das edificações; e NBR 16280 (ABNT, 2014), que trata sobre reformas em edificações.

Segundo Oliveira (2013), as inspeções devem ser realizadas por profissionais habilitados, sendo capazes de transmitir as informações técnicas necessárias para cada caso e realizar as inspeções de forma segura, minimizando os riscos de acidentes de trabalho. Em contrapartida, as inspeções físicas geralmente não são realizadas por profissionais habilitados na área de Patologia, transformando a inspeção em uma avaliação e resultando em diagnósticos imprecisos.

Para a realização da inspeção de fachadas, a metodologia mais conhecida é da inspeção física, onde o profissional vai até o problema com o auxílio de um equipamento de movimentação vertical externa. Entretanto, o difícil acesso às fachadas, com a necessidade do uso de andaimes ou plataformas elevatórias e o deslocamento de funcionários, eleva o custo para a realização das inspeções físicas, sendo esse um dos principais motivos da baixa periodicidade deste serviço.

Com o avanço da tecnologia, há um novo método de inspeção conhecido como inspeção virtual, onde utiliza-se um veículo aéreo não tripulado com uma câmera de alta resolução que capta imagens aéreas (drone), podendo ser realizado por um único profissional, estático em solo, apenas manuseando o equipamento.

A utilização da técnica de inspeção virtual pode diminuir os custos anteriormente citados na inspeção física e incentivar a realização mais frequente de inspeções, que por sua vez podem antecipar a necessidade de intervenção preventiva ou corretiva, evitando inúmeros acidentes e/ou danos.

Este estudo refere-se à comparação da eficiência da inspeção virtual com a inspeção física em fachadas com diferentes tipos de revestimentos e à verificação da viabilidade econômica.

\section{ESTUDO DE CASO}

A edificação definida como objeto de estudo encontra-se na cidade de Navegantes, litoral do estado de Santa Catarina (Figura 1). A escolha do empreendimento, em parceria com uma construtora local, ocorreu devido a necessidade de realização de uma inspeção nas fachadas norte e oeste após a detecção da presença de manifestações patológicas de origem externa em unidades autônomas.

O empreendimento totaliza $1.539,50 \mathrm{~m}^{2}$ de uso residencial composto por cinco pavimentos, sendo um pavimento garagem e quatro pavimentos tipo com quatro apartamentos por andar. A data de entrega foi 12 de agosto de 2015. 


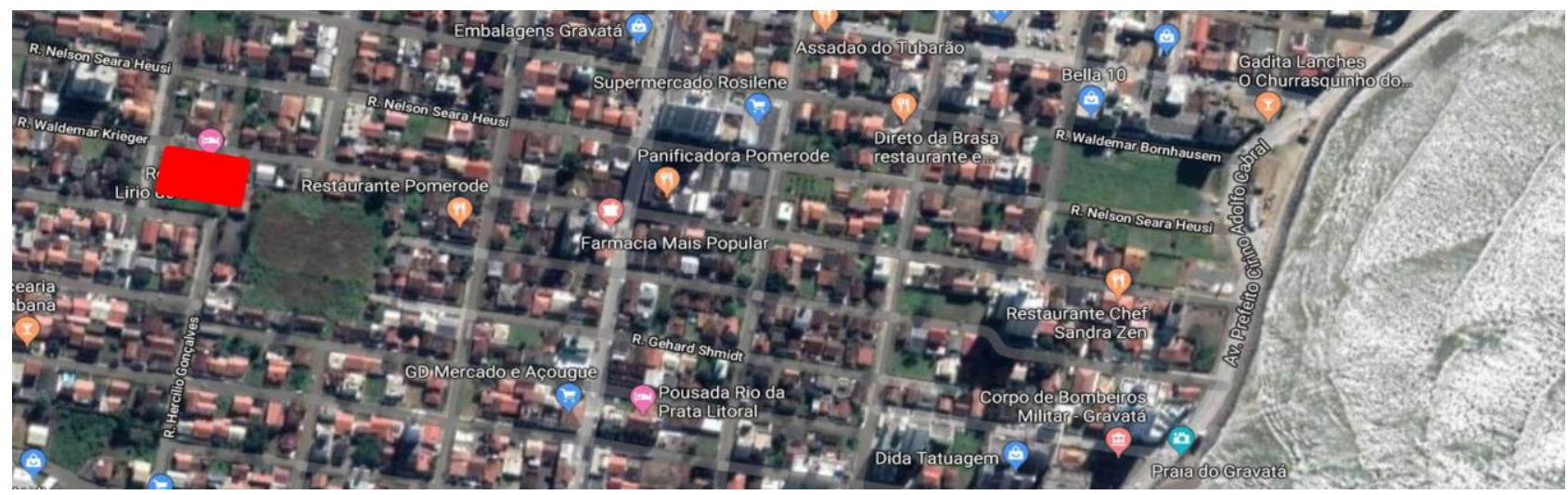

Figura 1: Localização da edificação em estudo.

Fonte: Google Maps, 2017. Adaptado.

Para a inspeção das fachadas fez-se a divisão em cinco panos, conforme demarcados na Figura 2. Em vermelho, foram definidos quatro panos idênticos de revestimento com pintura, com dimensões de 2,20 metros de altura por 9,30 metros de comprimento cada, obedecendo as limitações já presentes na edificação (panos 01 até 04). Já em azul, definiu-se um pano único de revestimento cerâmico com pastilhas de $5 \times 5 \mathrm{~cm}$ (pano 05).

Com a intenção de auxiliar a marcação das manifestações patológicas observadas durante as inspeções, elaborou-se um croqui das fachadas da edificação (Figura 2). Dessa maneira, o vistoriador pôde marcar o local exato onde foi identificado o dano e também pôde dimensionar aproximadamente a área que esse ocupa na fachada. Ao final das inspeções foi possível realizar uma consideração global da situação em que se encontravam os panos analisados.

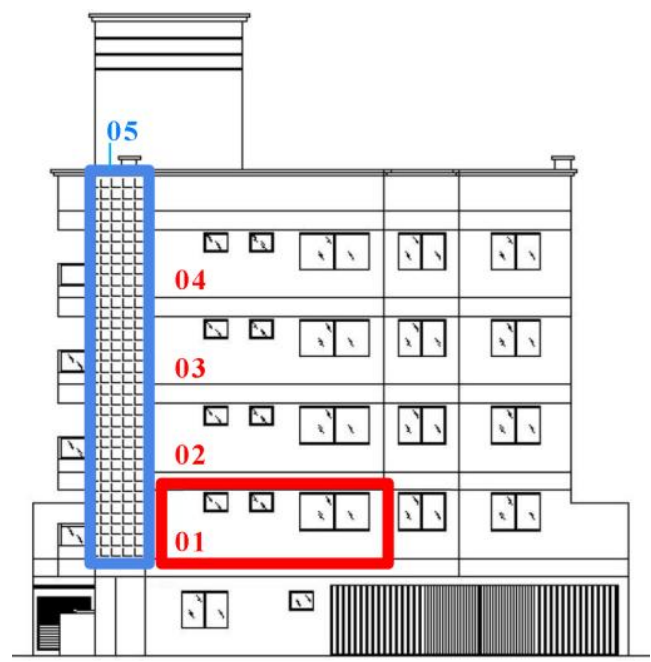

Figura 2: Croqui da edificação para a realização das inspeções.

A primeira inspeção realizada foi a física, a qual se deu através da utilização de um caminhão guindaste com um cesto (Figura 3-a). Esse tipo de equipamento de movimentação vertical externa dificultou a locomoção do vistoriador, que não obteve uma posição favorável para a marcação das manifestações patológicas no croqui e para retirada de fotos.

Devido a falta de precisão na inspeção física inicial, realizou-se uma segunda inspeção com a utilização de cadeira suspensa por corda (Figura 3-b). O uso desse tipo de equipamento facilitou a locomoção do vistoriador, tornando a inspeção mais abrangente, sendo possível analisar toda a área programada.

Através do auxílio de instrumentos, como o fissurômetro, e com o croqui em mãos, o vistoriador executou sucessivas descidas, em ordem pré-programada, verificando as manifestações patológicas existentes e registrando-as no croqui e através da retirada de fotos.

Nessa vistoria foi possível identificar a espessura real das fissuras encontradas (Figura 3-c), assim como realizar testes de percussão na área com revestimento cerâmico (Figura 3-d). 

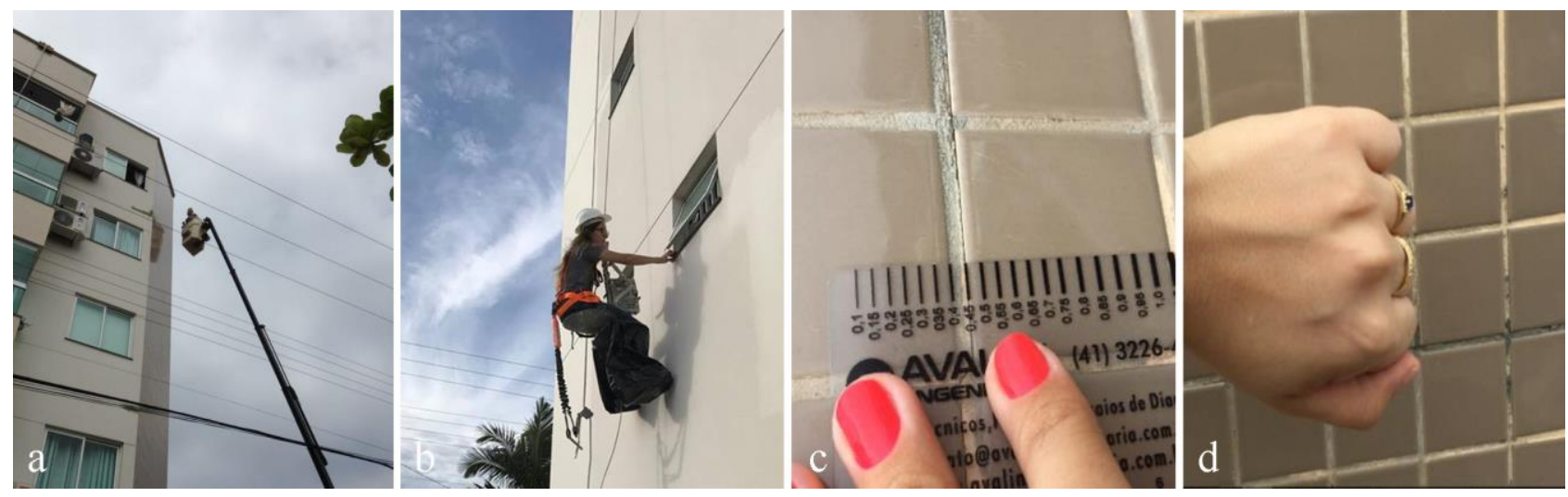

Figura 3: Inspeção física.

Após finalizar a inspeção física, realizou-se a inspeção virtual com o auxílio de um equipamento com câmera de alta resolução que capta imagens aéreas. O drone utilizado foi o modelo PHANTOM 3 ADVANCED de 2,7K vídeo e câmera com 12.0 mega pixels com o auxílio de um celular acoplado no controle, onde o vistoriador observava as imagens capturadas em tempo real (Figura 4-a). Tal caraterística permitiu movimentar o aparelho e registrar as imagens através de fotos com o ângulo e distância desejados. As fotos permeneceram salvas no celular utilizado para a inspeção.

A primeira inspeção virtual foi realizada por um profissional da área de vendas de imagens aéreas para o mercado imobiliário, ou seja, um controlador sem experiência na área de inspeção predial (Figura 4-b). Devido a velocidade acelerada dos vídeos e da grande distância da câmera do drone com relação à fachada, as imagens geradas não foram suficientes para a identificação das manifestações patológicas.

Em consequência do modo como o drone foi operado, realizou-se uma segunda inspeção virtual por um controlador técnico com experiência na área de Patologia (Figura 4-c). As fachadas foram avaliadas utilizando os mesmos princípios da inspeção física, realizando o registro através de fotografias e vídeos das regiões onde havia a suspeita da presença de danos para posterior análise das imagens com o auxílio da ferramenta computacional Windows Movie Maker. Esse programa possibilitou aproximar as imagens e modificar a velocidade dos vídeos capturados, sendo possível analisá-los quantas vezes se fizesse necessário.

Com base nos registros digitais pôde-se transcrever para o croqui de inspeção as manifestações patológicas observadas.

É importante salientar que houve a necessidade de operar o drone com cautela e baixa velocidade por conta das rajadas de vento e da proximidade do equipamento da edificação. Uma limitação observada está relacionada ao ângulo de inclinação da câmera do drone $\left(90^{\circ}\right)$ que acabou impossibilitando a inspeção em pingadeiras, balanços ou qualquer tipo de estrutura que necessitava ser visualizada no sentido de baixo para cima (Figura 4-d).
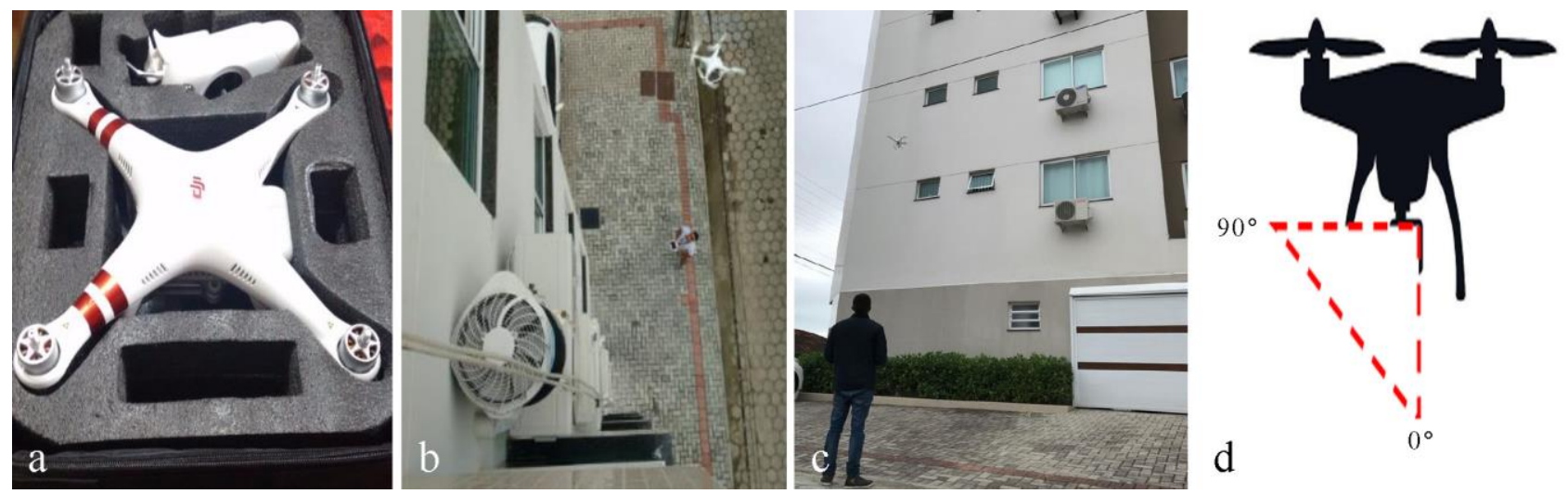

Figura 4: Vistoria virtual.

\section{ANÁLISE DOS RESULTADOS}

As manifestações patológicas observadas nas inspeções física e virtual são apresentadas nos croquis da Figura 5 e identificadas com base na numeração da Tabela 1. 

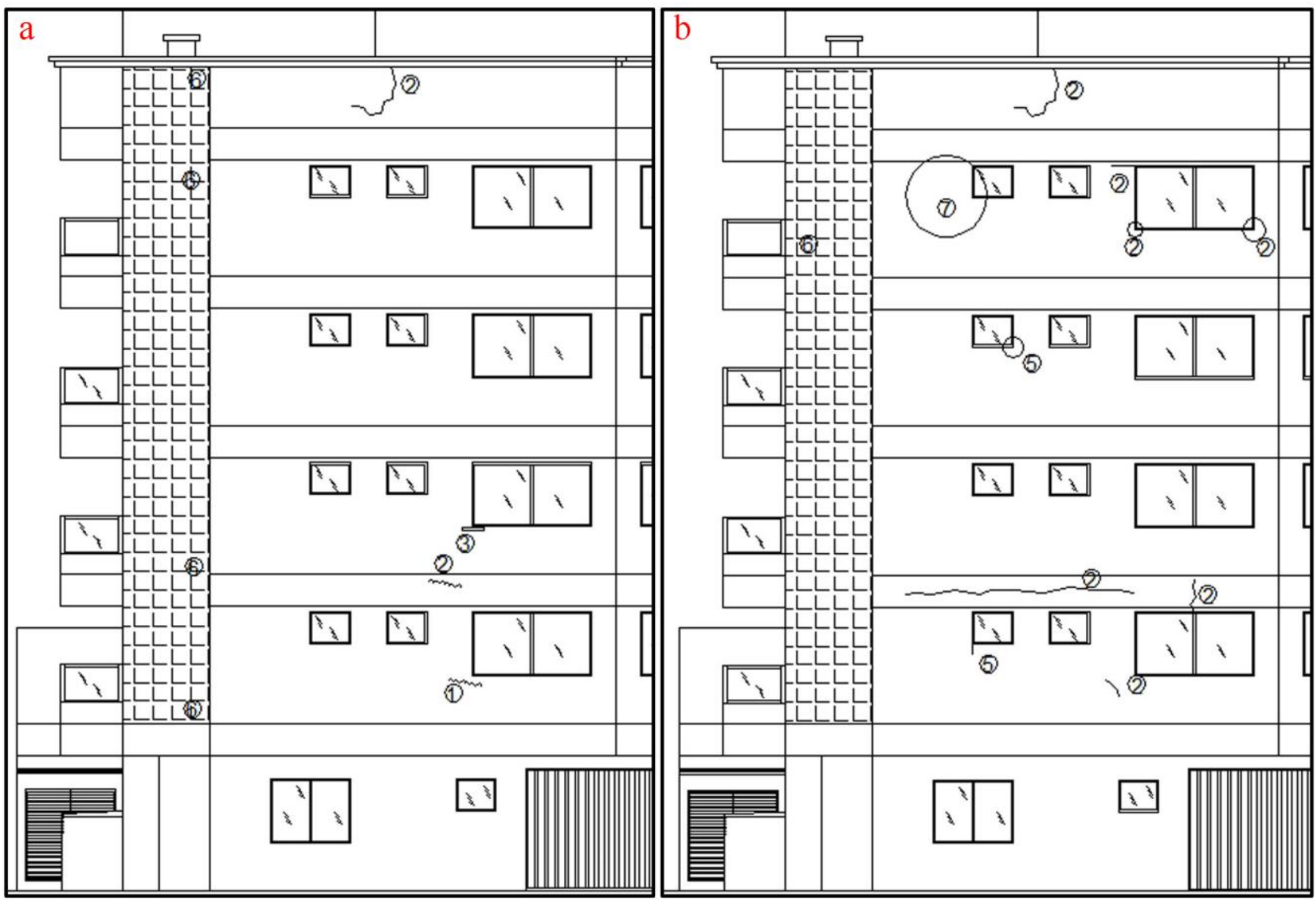

Figura 5: Croquis das manifestações patológicas observadas nas inspeções física (a) e virtual (b).

Tabela 1: Identificação das manifestações patológicas.

\begin{tabular}{c|c}
\hline Número & Manifestação patológica \\
\hline 1 & Fissura refeita pintura \\
\hline 2 & Fissura com abertura \\
\hline 3 & Silicone descolado \\
\hline 4 & Área sem pintura - reboco exposto \\
\hline 5 & Umidade \\
\hline 6 & Fissura em revestimento cerâmico \\
\hline 7 & Diferença na coloração do revestimento \\
\hline
\end{tabular}

Na Tabela 2 têm-se caracterizadas algumas manifestações patológicas observadas nas inspeções física e virtual. 
Tabela 2: Caracterização de manifestações patológicas observadas nas inspeções física e virtual.

\begin{tabular}{|c|c|c|}
\hline $\begin{array}{c}\text { Manifestação } \\
\text { patológica }\end{array}$ & Caracterização & Figura \\
\hline $\begin{array}{l}\text { Fissura na } \\
\text { platibanda }\end{array}$ & $\begin{array}{l}\text { Abertura relativamente grande de fácil identificação pelos dois } \\
\text { métodos de inspeção utilizados, não sendo possível visualizar o } \\
\text { dano do térreo da edificação sem a utilização de equipamentos. }\end{array}$ & \\
\hline $\begin{array}{l}\text { Fissura na } \\
\text { junta de } \\
\text { dilatação }\end{array}$ & $\begin{array}{l}\text { Através da inspeção virtual não foi possível obter clareza se o } \\
\text { dano era no revestimento de argamassa ou no selante. }\end{array}$ & \\
\hline $\begin{array}{l}\text { Diferença na } \\
\text { coloração do } \\
\text { revestimento }\end{array}$ & $\begin{array}{l}\text { Não é uma manifestação patológica, mas através das duas } \\
\text { inspeções foi possível identificar a diferença na coloração da tinta } \\
\text { utilizada no revestimento. Essa anomalia pode ser explicada por } \\
\text { reparo localizado realizado pela construtura, como também pode } \\
\text { ser um ponto de infiltração ou escorrimento de água. }\end{array}$ & \\
\hline $\begin{array}{l}\text { Fissuras em } \\
\text { revestimentos } \\
\text { cerâmico e de } \\
\text { pintura }\end{array}$ & $\begin{array}{l}\text { Para a identificação das manifestações patológicas nos } \\
\text { revestimentos cerâmicos com o auxílio de drone teve-se maior } \\
\text { dificuldade se comparado à inspeção física. Apenas pontos } \\
\text { críticos como o observado na Figura ao lado foram de fácil } \\
\text { visualização com o equipamento virtual. }\end{array}$ & \\
\hline $\begin{array}{l}\text { Mancha de } \\
\text { umidade em } \\
\text { revestimento }\end{array}$ & $\begin{array}{l}\text { A inspeção virtual possibilitou fácil identificação dos pontos de } \\
\text { escorrimento, umidade, fungos e eflorescência devido a vantagem } \\
\text { de visualizar inúmeras vezes as imagens capturadas. }\end{array}$ & \\
\hline $\begin{array}{l}\text { Trincas na } \\
\text { parte inferior } \\
\text { das janelas }\end{array}$ & $\begin{array}{l}\text { A inspeção física com a utilização de cadeira suspensa por corda, } \\
\text { por questões de segurança, normalmente não é realizada no } \\
\text { alinhamento das janelas, dessa forma fissuras existentes podem } \\
\text { passar despercebidas. Já a inspeção realizada com o auxílio de } \\
\text { plataformas elevatórias, caminhão guindaste ou com drone pode } \\
\text { eliminar tal característica negativa. }\end{array}$ & 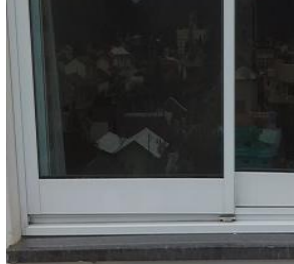 \\
\hline
\end{tabular}

Com os croquis das inspeções física e virtual preenchidos e identificadas as manifestações patológicas, fez-se o cálculo para avaliação do desempenho das inspeções conforme a metodologia proposta por Taguchi, onde os danos foram classificados de acordo com o estado de conservação para posterior contabilização de um valor final para cada pano estudado.

As Tabelas 3 e 4 apresentam os dados coletados nas inspeções física e virtual, respectivamente, onde:

Bi - Valor básico associado ao tipo de dano i;

K1i - Fator da importância do elemento de vedação;

$\mathrm{K} 2 \mathrm{i}$ - Fator indicativo da intensidade do dano i;

K3i - Fator indicativo da extensão do dano i;

K4i - Fator indicativo da urgência de intervenção para o dano i.

Para a inspeção física encontrou-se apenas dois tipos de manifestações patológicas nos cinco panos de fachada analisados. Com exceção de um dano com classificação de severidade de média dimensão no pano 05, todos os outros foram classificados como baixa severidade e existentes em poucos locais (Tabela 3). 
Também pôde-se observar, levando em consideração o valor K3i, que somento no pano 05 o dano compreendeu uma área maior se comparado aos outros panos em análise. Não foi encontrada nenhuma manifestação patológica no pano 03 (Tabela 3).

Com relação a inspeção virtual foi observado um dano no pano 03 e um dano adicional no pano 04 (Tabela 4).

Tabela 3: Inspeção física - Análise das manifestações patológicas.

\begin{tabular}{c|c|c|c|c|c|c|c|c|c}
\hline \multirow{2}{*}{ LOCAL } & \multirow{2}{*}{ TIPO DE DANO } & $\begin{array}{c}\text { CLASSE DE } \\
\text { SEVERIDADE }\end{array}$ & $\mathbf{B i}$ & $\mathbf{K 1 i}$ & $\mathbf{K 2 i}$ & $\mathbf{K 3 i}$ & $\mathbf{K} 4 \mathbf{i}$ & $\mathbf{I P}$ & $\mathbf{M} M$ \\
\hline Pano 01 & Fissura & 1 & 2 & 1 & 0,5 & 0,5 & 2 & 1 & 1 \\
\hline \multirow{2}{*}{ Pano 02 } & Fissura & 1 & 2 & 1 & 0,5 & 0,5 & 2 & 1 & 1,5 \\
\cline { 2 - 11 } & Umidade & 1 & 1 & 1 & 0,5 & 0,5 & 2 & 0,5 & - \\
\hline Pano 03 & - & - & - & - & - & - & - & - & - \\
\hline Pano 04 & Fissura & 1 & 2 & 1 & 0,5 & 0,5 & 3 & 1,5 & 1,5 \\
\hline Pano 05 & Fissura & 2 & 2 & 1 & 1 & 1 & 3 & 6 & 6 \\
\hline
\end{tabular}

Tabela 4: Inspeção virtual - Análise das manifestações patológicas.

\begin{tabular}{c|c|c|c|c|c|c|c|c|c}
\hline \multirow{2}{*}{ LOCAL } & TIPO DE DANO & $\begin{array}{c}\text { CLASSE DE } \\
\text { SEVERIDADE }\end{array}$ & $\mathbf{B i}$ & $\mathbf{K 1 i}$ & $\mathbf{K 2 i}$ & $\mathbf{K 3 i}$ & $\mathbf{K} 4 \mathbf{i}$ & $\mathbf{I P}$ & $\mathbf{M} \mathbf{\text { MM }}$ \\
\hline \multirow{2}{*}{ Pano 01 } & Mancha & 1 & 1 & 1 & 0,5 & 0,5 & 1 & 0,25 & - \\
\cline { 2 - 10 } & Fissura & 1 & 2 & 1 & 0,5 & 0,5 & 1 & 0,5 & 0,75 \\
\hline Pano 02 & Fissura & 2 & 2 & 1 & 1 & 0,5 & 2 & 2 & 2 \\
\hline Pano 03 & Mancha & 1 & 1 & 1 & 0,5 & 0,5 & 1 & 0,25 & 0,25 \\
\hline \multirow{2}{*}{ Pano 04 } & Mancha & 1 & 1 & 1 & 0,5 & 0,5 & 1 & 0,25 & - \\
\cline { 2 - 10 } & Fissura & 2 & 2 & 1 & 1 & 0,5 & 2 & 2 & 2,25 \\
\hline Pano 05 & Fissura & 2 & 2 & 1 & 1 & 1 & 3 & 6 & 6 \\
\hline
\end{tabular}

Tratando-se das Tabelas analisadas anteriormente, é possível verificar grande proximidade na quantidade e classificação das manifestações patológicas com relação aos cinco panos de fachada estudados. Para a inspeção virtual verificou-se um maior número de danos, mas com valores de referência menores.

Seguindo a recomendação do autor da metodologia de cálculo proposta, definiu-se uma parede referência para posterior comparação final dos resultados, a qual é estabelecida como sendo o pior caso possível para os cinco panos de fachada em análise.

A Tabela 5 relaciona as manifestações patológicas encontradas nas inspeções física e virtual com o pior estado possível dentro das pré-definições de Taguchi.

Tabela 5: Parede referência.

\begin{tabular}{c|c|c|c|c|c|c|c|c|c}
\hline \multirow{2}{*}{ LOCAL } & TIPO DE DANO & $\begin{array}{c}\text { CLASSE DE } \\
\text { SEVERIDADE }\end{array}$ & $\mathbf{B i}$ & $\mathbf{K 1 i}$ & $\mathbf{K 2 i}$ & $\mathbf{K 3 i}$ & $\mathbf{K 4 i}$ & $\mathbf{I P}$ & $\mathbf{M M}$ \\
\hline \multirow{2}{*}{$\begin{array}{c}\text { Pano de } \\
\text { referência }\end{array}$} & Umidade & 4 & 1 & 1 & 2 & 2 & 1 & 4 & - \\
\cline { 2 - 11 } & Mancha & 4 & 1 & 1 & 2 & 2 & 1 & 4 & - \\
\cline { 2 - 10 } & Fissura & 4 & 2 & 1 & 2 & 2 & 1 & 8 & 16 \\
\hline
\end{tabular}

Para comparar os resultados dos danos efetivos e dos danos da parede referência, determinou-se o índice de performance global (IPg) e com isso a classificação de cada pano para as inspeções física e virtual.

A identificação final da situação de cada pano com relação à deterioração e ao impacto que isso pode causar à segurança e salubridade do edifício analisado é dada através das classes I a VI (sem danos até crítico grau de deterioração). A parede referência apresentou o maior grau de deterioração, sendo classificada como classe VI.

Nas Tabelas 6 e 7 são apresentados os valores das inspeções física e virtual, assim como a classificação de cada pano de acordo com o seu valor de IPg baseado no pano de referência. 
Tabela 6: Classificação dos panos - Inspeção física.

\begin{tabular}{c|c|c|c|c|c|c|c|c}
\hline Elemento & K1m & Mm & Def. & K1m & Mm,ref. & Dref. & IPg & Classe \\
\hline Parede referência & 1 & 16 & - & 1 & 16 & - & 100,00 & - \\
\hline Pano 01 & 1 & 1 & 1 & 1 & 16 & 16 & 6,25 & II \\
\hline Pano 02 & 1 & 1,5 & 1,5 & 1 & 16 & 16 & 9,375 & II \\
\hline Pano 03 & 1 & 0 & 0 & 1 & 16 & 16 & 0 & I \\
\hline Pano 04 & 1 & 1,5 & 1,5 & 1 & 16 & 16 & 9,365 & II \\
\hline Pano 05 & 1 & 6 & 6 & 1 & 16 & 16 & 37,5 & VI \\
\hline
\end{tabular}

Tabela 7: Classificação dos panos - Inspeção virtual.

\begin{tabular}{c|c|c|c|c|c|c|c|c}
\hline Elemento & K1m & Mm & Def. & K1m & Mm,ref. & Dref. & IPg & Classe \\
\hline Parede referência & 1 & 16 & - & 1 & 16 & - & 100,00 & - \\
\hline Pano 01 & 1 & 0,75 & 0,75 & 1 & 16 & 16 & 4,69 & II \\
\hline Pano 02 & 1 & 2 & 2 & 1 & 16 & 16 & 12,50 & III \\
\hline Pano 03 & 1 & 0,25 & 0,25 & 1 & 16 & 16 & 1,56 & I \\
\hline Pano 04 & 1 & 2,25 & 2,25 & 1 & 16 & 16 & 14,06 & III \\
\hline Pano 05 & 1 & 6 & 6 & 1 & 16 & 16 & 37,5 & VI \\
\hline
\end{tabular}

Com base nos valores de IPg e o resultado da classificação dos panos, elaborou-se um gráfico com o intuito de comparar cada pano analisado com cada método de inspeção (Figura 6).

Através da Figura 6 é possível verificar a semelhança entre os resultados das inspeções física e virtual, a proximidade na identificação das classes e consequentemente da eficiência das inspeções. Percebe-se a proximidade dos resultados nos panos de fachada 01,02 e 04 e a equivalência dos resultados no pano 05.

Apenas na inspeção virtual foi identificada uma manifestação patológica no pano 03, onde através de registros fotográficos realizados pelo drone constatou-se que o dano era de baixa gravidade e de pequena dimensão. Provável razão pela qual o dano não foi identificado na inspeção física.

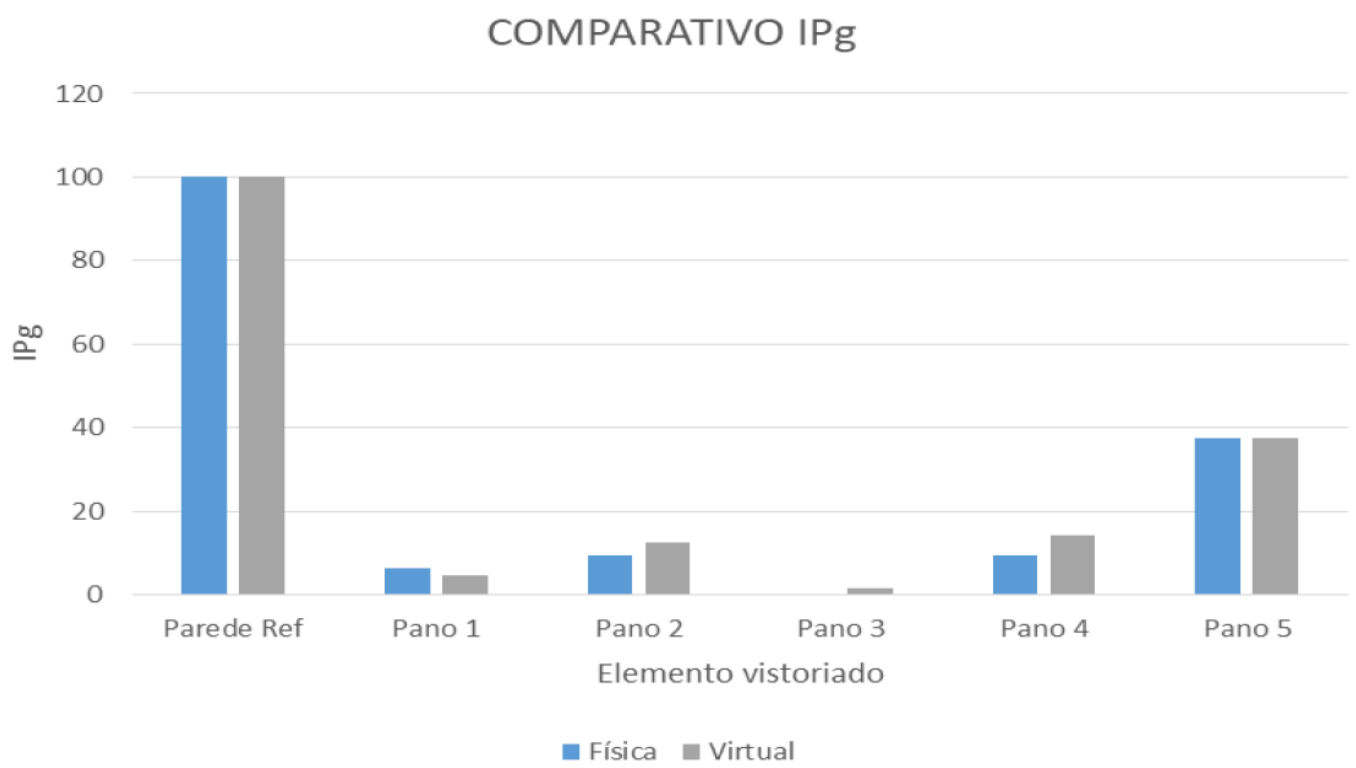

Figura 6: Gráfico comparativo Ipg (índice de performance global) para as inspeções física e virtual.

Considerando a viabilidade financeira através da realização de orçamento com empresa especializada em aluguel de equipamentos para a construção civil na região de Navegantes (SC), têm-se os valores médios de locação a seguir. 
Tabela 8: Orçamentos andaime.

\begin{tabular}{l|c|c|c|c}
\multicolumn{1}{c|}{ Equipamento } & Quant. & Período & Valor unitário & Valor total \\
\hline Andaime suspenso mecânico - 3 mts & 1 & 07 dias & $\mathrm{R} \$ 425,04$ & $\mathrm{R} \$ 425,04$ \\
\hline Andaime suspenso elétrico - 3 mts & 1 & 07 dias & $\mathrm{R} \$ 1.013,06$ & $\mathrm{R} \$ 1.013,06$ \\
\hline Andaime suspenso mecânico - 3 mts & 1 & 30 dias & $\mathrm{R} \$ 607,20$ & $\mathrm{R} \$ 607,20$ \\
\hline Andaime suspenso elétrico - 3 mts & 1 & 30 dias & $\mathrm{R} \$ 1.448,70$ & $\mathrm{R} \$ 1.448,70$ \\
\hline $\begin{array}{l}\text { Troca de lugar do andaime suspenso } \\
\text { (desmontagem e montagem) }\end{array}$ & - & - & $\mathrm{R} \$ 300,00$ & - \\
\hline $\begin{array}{l}\text { Visita técnica / manutenção em caso de } \\
\text { divergência do cliente }\end{array}$ & - & - & $\mathrm{R} \$ 90,00$ & - \\
\hline
\end{tabular}

Fonte: Construrenova (2017).

Para o aluguel do drone, têm-se: duzentos reais (R\$ 200,00) para duas horas de cobertura de eventos ou fotos, considerando um número ilimitado de fotos e vídeos dentro do período determinado, e voo realizado pelo proprietário do equipamento.

Considerando a aquisição de um drone há uma grande variação de valores no mercado devido a diferença de marca, modelo e qualidade fotográfica. O preço médio de mercado no Brasil do modelo de equipamento utilizado neste estudo é de dois mil e trezentos reais até dois mil e setecentos reais ( $\mathrm{R} \$ 2.300,00$ até $\mathrm{R} \$ 2.700,00)$, considerando o aparelho sem os acessórios extras.

Deve-se levar em consideração ainda a diária da mão de obra para a inspeção da fachada (Tabela 9).

Tabela 9: Comparativo de custos.

\begin{tabular}{l|c|c|c}
\hline & $\begin{array}{c}\text { Física } \\
\text { Andaime suspenso }\end{array}$ & $\begin{array}{c}\text { Virtual } \\
\text { Aluguel }\end{array}$ & $\begin{array}{c}\text { Virtual } \\
\text { Aquisição }\end{array}$ \\
\hline Equipamento & $\begin{array}{c}\mathrm{R} \$ 425,07 \\
\text { dias (aluguel) }\end{array}$ & $\begin{array}{c}\mathrm{R} \$ 200,00 \\
2 \text { horas (aluguel) }\end{array}$ & $\mathrm{R} \$ 2.500,00$ \\
\hline Mão de obra & $\mathrm{R} \$ 200,00$ \\
01 dia & Incluso & - \\
\hline Movimentação equipamento & $\mathrm{R} \$ 300,00$ & - & - \\
\hline $\begin{array}{l}\text { Visita técnica (garantia segurança } \\
\text { equipamento) }\end{array}$ & $\mathrm{R} \$ 90,00$ & - & - \\
\hline TOTAL & $\mathrm{R} \$ 1.015,07$ & $\mathrm{R} \$ 200,00$ & $\mathrm{R} \$ 2.500,00$ \\
\hline
\end{tabular}

Fonte: Construrenova (2017)

Para a realização da inspeção física deste estudo, utilizou-se o andaime mais barato e com apenas uma movimentação. Já para a inspeção virtual, duas horas foram suficientes para a realização das fotos e vídeos das fachadas, sendo que apenas posteriormente foram elaboradas as análises.

Ao comparar a opção de utilização do drone com qualquer aluguel de balancim para a realização da inspeção, a análise virtual torna-se mais econômica.

É possível afirmar que a aquisação do drone torna-se viável para a realização de inspeção virtual em empreendimentos de diversos tamanhos, uma vez que o investimento realizado é único e o uso pode ser contínuo, não dependendo de outros profissionais e equipamentos. Para essa opção e necessário um investimento médio de dois mil e quinhentos reais $(\mathrm{R} \$ 2.500,00)$ para o equipamento básico.

Levando em consideração o estudo realizado, elaborou-se uma Tabela que apresenta as principais vantagens e desvantagens para os métodos de inspeção física e virtual. 
Tabela 10: Vantagens e desvantagens para as inspeções física e virtual.

\begin{tabular}{|c|c|c|}
\hline Itens em análise & Inspeção física & Inspeção virtual \\
\hline Menor custo (sem aquisição do equipamento) & $x$ & $\checkmark$ \\
\hline Maior agilidade na vistoria & $x$ & $\checkmark$ \\
\hline Segurança & X & $\sqrt{ }$ \\
\hline Banco de dados & $x$ & $\checkmark$ \\
\hline Trabalho em grandes alturas & $\checkmark$ & X \\
\hline Ensaio físico & $\checkmark$ & $x$ \\
\hline Clima e tempo & $x$ & $x$ \\
\hline Inspeção por profissional habilitado & $x$ & $\checkmark$ \\
\hline Limitação em ângulos maiores que $90^{\circ}$ & $\checkmark$ & $x$ \\
\hline Repetibilidade da análise & $x$ & $\checkmark$ \\
\hline Interferência da paleta de cores do revestimento & $x$ & $\checkmark$ \\
\hline Inspeção com maior confiabilidade em revestimento com pintura & $x$ & $\checkmark$ \\
\hline Inspeção com maior confiabilidade em revestimento cerâmico & $\checkmark$ & $x$ \\
\hline
\end{tabular}

\section{CONSIDERAÇÕES FINAIS}

Com a finalidade de contribuir para a realização de inspeções periódicas nas fachadas de edificações e consequentemente com a prevenção de manifestações patológicas através da realização de manutenções preventivas, fez-se o comparativo da eficiência da inspeção virtual com a inspeção física em fachadas com diferentes tipos de revestimentos e também a verificação da viabilidade econômica.

Os resultados deste estudo correspondem à cinco panos de fachada de empreendimento localizado na cidade de Navegantes (SC), portanto não devem ser generalizados para outras condições sem o estudo prévio.

Com base nos resultados das inspeções física e virtual do estudo de caso e na análise realizada a partir da metodologia proposta por Taguchi, pode-se afirmar que as imagens realizadas pelo drone apresentaram qualidade suficiente para a identificação e análise das manifestações patológicas, inclusive as de menor tamanho. Além disso, com o auxílio de softwares é possível medir o comprimento e a espessura dos danos como fissuras, detalhando-os com maior precisão. Entretanto, para danos como desplacamentos e deficiências no assentamento de placas cerâmicas, embora não houvesse nos panos em estudo, pode-se afirmar que não é possível apresentar conclusões, devido a necessidade de realização de inspeção física (teste de percussão).

Apesar da dificuldade de pilotar o drone em dias de rajadas de vento devido a proximidade necessária da edificação, precisando controlá-lo com cautela e baixa velocidade, e da limitação do ângulo de inclinação da câmera do equipamento $\left(90^{\circ}\right)$, a inspeção virtual apresentou um bom resultado quando realizada por um profissional especializado na área de Patologia. É valido destacar que alterações climáticas também podem influenciar negativamente a inspeção física, havendo a perda de desempenho do vistoriador e/ou a necessidade de suspensão das atividades.

Tratando-se da eficiência e precisão dos resultados, os dois tipo de inspeção se assemelharam. Mas, a inspeção virtual apresentou vantagens, como: revisão das imagens, devido os registros serem realizados em vídeo; rapidez na realização da inspeção; e maior viabilidade econômica, devido o menor custo de aluguel de equipamentos se relacionado às horas trabalhadas. Então, pode-se considerar a inspeção virtual com o auxílio de drone a mais eficiente.

\section{REFERÊNCIAS}

ALVES, S. M. A. WWW.PATORREB.COM - Um contributo para a sistematização do conhecimento da patologia da construção. Faculdade de Engenharia da Universidade do Porto, 2008.

ANTUNES, G. R. Estudo de manifestações patológicas em revestimento de fachada em Brasília: sistematização da incidência de casos. Dissertação de Mestrado, Universidade Federal de Brasília, 2010.

ASSOCIAÇÃO BRASILEIRA DE NORMAS TÉCNICAS. NBR 13752: Perícias de engenharia na construção civil. Rio de Janeiro, 1996.

NBR 15575-1: Edifícios habitacionais - Desempenho - Parte 1: Requisitos gerais. Rio de Janeiro, 2013. 
NBR 16280: Reforma em edificações - Sistema de gestão de reformas - Requisitos. Rio de Janeiro, 2014.

BAUER, E. Resistência a Penetração da Chuva em Fachadas de Alvenaria de Materiais Cerâmicos - Uma Análise de Desempenho. Dissertação de Mestrado, Universidade Federal do Rio Grande do Sul, Porto Alegre, 1987.

BAUER, L. A. F. Materiais de construção. 5. ed. Rio de Janeiro: LTC, 1994.

CAMPANTE, E. F. Metodologia de diagnóstico, recuperação e prevenção de manifestações patológicas em revestimentos cerâmicos de fachadas. Tese de Doutorado, Escola Politécnica da Universidade de São Paulo, Departamento de Engenharia de Construção Civil, São Paulo, 2001.

CARASEK, H. Patologia das argamassas de revestimento. Materiais de Construção e Princípios de Ciência e Engenharia de Materiais. IBRACON, São Paulo, v. 1, 2007.

CEOTTO, L. H.; Banduk, R. C.; Nakakura, E. H. Revestimentos de Argamassas: boas Práticas em projeto, execução e avaliação. Porto Alegre: Prolivros,2005. (Recomendações Técnicas HABITARE, 1).

CONSOLI, O. J. Análise da durabilidade dos componentes de fachadas de edifícios, sob a ótica do projeto arquitetônico. Dissertação de Mestrado, Universidade Federal de Santa Catarina, Florianópolis, 2006.

FLORES, I. Metodologia de avaliação do desempenho em serviço de fachadas rebocadas na óptica da manutenção predictiva. Dissertação de Doutorado. Instituto Superior Técnico, Universidade Técnica de Lisboa, Lisboa, 2009.

GASPAR, P. Vida útil das construções: Desenvolvimento de uma metodologia para a estimativa da durabilidade de elementos da construção. Aplicação a rebocos de edifícios correntes. Dissertação de Doutorado, Instituto Superior Técnico, Universidade Técnica de Lisboa, Lisboa, 2009.

GUIMARÃES, L. E. Avaliação comparativa de grau de deterioração de edificações - Estudo de caso: prédios pertencentes à Universidade Federal de Goiás. Dissertação de Mestrado, Universidade Federal de Goiás, Goiânia, 2003.

GOLDBERG, R. P. Direct adhered ceramic tile, stone and thin brick facades. Technical Design Manual. LATICRETE International, Inc. USA. 1998.

HELENE, P. R. L. Manual para reparo, reforço e proteção de estruturas de concreto. 2. ed. São Paulo: PINI, 1992.

INSITUTO BRASILEIRO DE AVALIAÇÕES E PERÍCIAS DE ENGENHARIA. Inspeção predial: a saúde dos edifícios. 2 ed. São Paulo: IBAPE, 2015.

INSITUTO BRASILEIRO DE AVALIAÇÕES E PERÍCIAS DE ENGENHARIA. Inspeção predial no Senado da República - Agora é projeto lei. Rio Grande do Sul: 2012.

INSITUTO BRASILEIRO DE AVALIAÇÕES E PERÍCIAS DE ENGENHARIA. Norma de Inspeção Predial. São Paulo, 2009. Disponível em <http://www.ibape-sp.org.br/arquivos/CARTILHA-Inspecao-predial-a-saude-dosedif\%C3\%ADcios.pdf> Acesso em 14 Jun.16.

INSITUTO BRASILEIRO DE AVALIAÇÕES E PERÍCIAS DE ENGENHARIA. Norma de Inspeção Predial. São Paulo, 2011. Disponível em 〈http://www.ibape-sp.org.br/arquivos/norma_de_inspecao_predial.pdf〉. Acesso em 14 Jun.16.

IOSHIMOTO, E. Incidência de manifestações patológicas em edificações habitacionais. In: Tecnologia de edificações. São Paulo: PINI, IPT - Instituto de Pesquisas Tecnológicas do Estado de São Paulo, Coletânea de trabalhos da Div. de Edificações do IPT,1988.

LICHTENSTEIN, N. B. Patologia das construções: procedimento para diagnóstico e recuperação, Boletim Técnico: BT/PCC/06. São Paulo, EPUSP, 1985.

LUZ, M. A. Manifestações Patológicas Em Revestimentos Cerâmicos de Fachada em Três Estudos de Caso na Cidade de Balneário Camboriú. Dissertação de Mestrado, Universidade Federal de Santa Catarina, 2004. 
OLIVEIRA, C. S. P. Análise Crítica de Experiências e Discussão de Estratégias para Implantação de Leis de Inspeção de Elementos de Fachadas. Tese de Doutorado. Universidade Federal do Rio Grande do Sul. Porto Alegre: 2013.

PAES, I. N. L; CARASEK, H. Desempenho de argamassas de rejuntamento no sistema de revestimento cerâmico, Associação Nacional de Tecnologia do Ambiente Construído - ANTAC, Porto Alegre, v.2, 2002.

PEREZ, A. R. Umidade nas edificações. Dissertação de mestrado em Engenharia Civil, Escola Politécnica da Universidade de São Paulo, São Paulo, Brasil, 1986.

SABBATINI, F. H.; BARROS, M. M. S. B. Produção de revestimentos cerâmicos para paredes de vedação em alvenaria: diretrizes básicas. Escola Politécnica da USP, São Paulo, 2001.

SARAIVA, A. G. Contribuição ao Estudo de Tensões de Natureza Térmica em Sistemas de Revestimento Cerâmico de Fachada. Dissertação de Mestrado, Universidade de Brasília, Brasília, 1998.

SILVA, A. F. Manifestações patológicas em fachadas com revestimentos argamassados: estudo de caso em edifícios em Florianópolis. Florianópolis, 2007.

SILVA, MARIA DE NAZARÉ BATISTA DA. Avaliação Quantitativa da Degradação e Vida Útil de Revestimentos de Fachada - Aplicação ao Caso de Brasília/DF [Distrito Federal] 2014.

THOMAZ, E. Trincas em edifícios: causas, prevenção e recuperação. 1 ed. São Paulo: PINI/EPUSP/IPT, 1989. 